\title{
Right ventricular wall motion abnormalities $n$ Thalassemia Major patients
}

\author{
Antonella Meloni ${ }^{1 *}$, Vincenzo Positano ${ }^{1}$, Sabrina Armari ${ }^{2}$, Giuseppina Secchi ${ }^{3}$, Giancarlo Izzi ${ }^{4}$, Cristina Salvatori ${ }^{1}$, \\ Claudio Ascioti ${ }^{5}$, Gennaro Restaino ${ }^{6}$, Massimo Lombardi ${ }^{1}$, Alessia Pepe ${ }^{1}$ \\ From 17th Annual SCMR Scientific Sessions \\ New Orleans, LA, USA. 16-19 January 2014
}

\section{Background}

Movement abnormalities in the left ventricle (LV) were shown to be not really frequent in thalassemia major (TM) patients but they were associated with age, myocardial iron overload, LV dilation and dysfunction, and myocardial fibrosis. No data are available about the prevalence and the correlates of right ventricular (RV) wall motion abnormalities. This study investigated the relationship between RV and LV motion abnormalities and between RV motion and function in a large cohort of well-treated TM patients.

\section{Methods}

CMR was performed in 1092 TM patients (537 male; $30.6 \pm 8.5$ years) enrolled in the Myocardial Iron Overload in Thalassemia (MIOT) Network. Cine images were acquired to evaluate wall motion and to quantify $\mathrm{RV}$ volumes and ejection fraction (EF).

\section{Results}

Abnormal motion of the LV was found in $66(6 \%)$ patients (60 hypokinetic and 6 dyskinetic). Abnormal motion of the RV was found in 35 (3.2\%) patients (29 hypokinetic, 5 dyskinetic and 1 akynetic). Abnormal LV motion was not correlated with abnormal RV motion. Seventeen patients showed movement abnormalities in both ventricles. The Table 1 shows the comparison between TM patients with normal and abnormal RV motion. Patients with abnormal RV motion were older and they were more frequently males. Right volumes indexed by body surface area (BSA) were significantly higher in patients with abnormal RV motion while the EF was significantly lower.

\section{Conclusions}

In TM patients movement abnormalities in the right ventricle were less frequent compared to the left ventricle, but were associated with age, sex, RV dilation and dysfunction.

\section{Funding}

The MIOT project receives "no-profit support" from industrial sponsorships (Chiesi Farmaceutici S.p.A. and ApoPharma Inc.). This study was also supported by: "Ministero della Salute, fondi ex art. 12 D.Lgs. 502/92 e

Table 1

\begin{tabular}{cccc}
\hline & $\begin{array}{c}\text { Abnormal } \\
\text { RV motion }\end{array}$ & Normal RV motion & P \\
\hline Age & $33.9 \pm 5.9$ & $30.5 \pm 8.6$ & 0.013 \\
\hline Sex (M/F) & $27 / 8$ & $510 / 547$ & 0.001 \\
\hline$R V$ end-diastolic volume index $(\mathrm{m} / \mathrm{m} / \mathrm{m})$ & $110.4 \pm 48.2$ & $83.4 \pm 19.2$ & $<0.0001$ \\
\hline$R V$ end-systolic volume index $(\mathrm{m} / \mathrm{m} 2)$ & $61.5 \pm 29.6$ & $32.5 \pm 11.4$ & $<0.0001$ \\
\hline$R V$ ejection fraction $(\%)$ & $44.9 \pm 10.1$ & $61.4 \pm 7.7$ & $<0.0001$ \\
\hline
\end{tabular}

${ }^{1}$ CMR Unit, Fondazione G.Monasterio CNR-Regione Toscana and Institute of

Clinical Physiology, Pisa, Italy

Full list of author information is available at the end of the article 
s.m.i., ricerca sanitaria finalizzata anno 2006" and "Fondazione L. Giambrone".

\section{Authors' details}

${ }^{1}$ CMR Unit, Fondazione G.Monasterio CNR-Regione Toscana and Institute of Clinical Physiology, Pisa, Italy. ${ }^{2}$ Reparto di Pediatria, Azienda Ospedaliera di Legnago U.O., Legnago, Italy. ${ }^{3}$ Servizio trasfusionale, Azienda USL no 1 , Sassari, Italy. ${ }^{4}$ Dipartimento Materno-Infantile U.O. Pediatria Oncoematologia, Azienda Ospedaliera di Parma, Parma, Italy. ${ }^{5}$ Struttura Complessa di Cardioradiologia, P.O. "Giovanni Paolo II", Lamezia Terme, Italy. 'Dipartimento di Radiologia, Università Cattolica del Sacro Cuore - Centro di Ricerca e Formazione ad Alta Tecnologia nelle Scienze Biomediche, Campobasso, Italy.

Published: 16 January 2014

doi:10.1186/1532-429X-16-S1-P257

Cite this article as: Meloni et al:: Right ventricular wall motion

abnormalities $\mathrm{n}$ Thalassemia Major patients. Journal of Cardiovascular

Magnetic Resonance 2014 16(Suppl 1):P257.

\section{Submit your next manuscript to BioMed Central} and take full advantage of:

- Convenient online submission

- Thorough peer review

- No space constraints or color figure charges

- Immediate publication on acceptance

- Inclusion in PubMed, CAS, Scopus and Google Scholar

- Research which is freely available for redistribution

Submit your manuscript at www.biomedcentral.com/submit
C Biomed Central 